\title{
Trabajando la Enfermería transcultural en el aula
}

\section{Working with transcultural nursing in the classroom}

ROCío DE DIEGO CORDERO,

ORCID: http://orcid.org/0000-0002-3453-003X

Universidad de Sevilla,

Departamento de Enfermería

rdediego2@us.es

Fecha de recepción: 14-06-2019.

Fecha de aceptación: 28-06-2019

DOI: http://dx.doi.org/10.12795/9788447221912.026

Pp.: 601-618 


\section{Resumen}

Se ha realizado un ciclo de mejora en el aula (CIMA) en la asignatura Historia, Teoría y Métodos de la Enfermería I de 10 Grado Enfermería, llevando a cabo sesiones en el trabajo en grupos pequeños (seminarios) donde se incrementó la participación del alumnado mediante la realización de esquemas de contenidos y la resolución de casos clínicos, incorporando además la evaluación de ideas previas que ayudaron a orientar las sesiones y material audiovisual para una mejor comprensión de los contenidos. Los resultados muestran la mejora en el aprendizaje en el grupo.

Palabras clave: Historia, teoría y métodos de la Enfermería I; Grado en Enfermería; Docencia Universitaria, Experimentación Docente Universitaria, Enfermería Transcultural

\section{Abstract}

There has been a cycle of improvement in the classroom (CIMA) in the subject History, Theory and Methods of Nursing I 1st Degree Nursing, conducting sessions in small group work (seminars) where the participation of students increased through the creation of content schemes and the resolution of clinical cases, also incorporating the evaluation of previous ideas that helped to guide the sessions and audiovisual material for a better understanding of the contents. The results show the improvement in learning in the group.

Keywords: History, theory and methods of Nursing I; Degree in Nursing; University Teaching, University Teaching Experimentation, Transcultural Nursing

Jornadas de Formación e Innovación Docente del Profesorado | № 2 (2019) Esta obra se distribuye con la licencia Creative Commons Reconocimiento-NoComercial-SinObraDerivada 4.0 Internacional (CC BY-NC-ND 4.0.) 


\section{Breve descripción del contexto}

La intervención se ha realizado con alumnado de la asignatura Historia, Teoría y Métodos de la Enfermería I, de 1er curso del Grado en Enfermería, durante el curso 2018/2019. Los contenidos teóricos de esta asignatura pretenden que el alumno:

- Identifique y relacione el concepto de salud y los cuidados, desde una perspectiva histórica comprendiendo la evolución del cuidado de enfermería.

- Explique desde una perspectiva ontológica y epistemológica, la evolución de los conceptos centrales que configuran la disciplina de enfermería.

- Identifique y diferencie desde una perspectiva ontológica y epistemológica los modelos teóricos más relevantes de la disciplina de enfermería.

A ello hay que unir las competencias trasversales que se pretenden adquieran con el curso de la misma, tales como el compromiso ético, la capacidad de crítica y autocrítica, la habilidad para trabajar de forma autónoma, la capacidad para aplicar la teoría a la práctica, la capacidad de aprender, la solidez en los conocimientos básicos de la profesión, las habilidades para trabajar en grupo y la capacidad de análisis y síntesis.

Se trata de un grupo de 12 estudiantes, de edades entre los 18 y los 20 años, procediendo en su totalidad del bachillerato. Trabajan divididos en 3 grupos de 4 estudiantes. El CIMA se llevó a cabo los días 13, 20 y 27 de Mayo de 2019 y tuvo una duración de cinco horas. 


\section{Diseño previo del CIMA}

\section{Mapa de contenidos y problemas}

La diversidad cultural es cada vez mayor y la inmigración hace que nos encontremos con un grupo de personas que posee unos conocimientos, creencias y pensamientos distintos de los demás relacionados con su cultura. Conceptos tan básicos como salud y enfermedad tienen distintas connotaciones dependiendo de la cultura (González, 2006). Como consecuencia, se producen barreras culturales que deben ser subsanadas por los enfermeros/as y para lograrlo, estos deben tener una formación específica en cuidados culturales y conocer un modelo basado en la transculturalidad (Castillo-Martínez y Fernández-Pujazón, 2015), de ahí la importancia de esta temática para el alumnado de enfermería.

Madeleine Leininger desarrolló la Teoría de Enfermería Transcultural. Defiende que la salud y los cuidados son elementos universales pero el modo en el que ellos se llevan a cabo y cómo son interpretados por la persona varía dependiendo de sus creencias. Influenciada por sus conocimientos en Antropología, a principios de la década de los sesenta definió la Enfermería Transcultural como: "el área de estudio y trabajo centrado en el cuidado basado en la cultura, creencias de salud o enfermedad, valores y prácticas de las personas, para ayudarlas a mantener o recuperar la salud, hacer frente a sus discapacidades o muerte". Los factores que influyeron en ella para defender la Enfermería Transcultural y argumentar su teoría sobre el Cuidado Cultural y Universal fueron: el aumento de la diversidad (étnica, racial, de género, edad...); la necesidad de que existan enfermeros/as que entiendan y respeten estas diferencias culturales; el conflicto cultural existente debido al abuso de la tecnología en salud, el efecto de los 
conflictos culturales en la salud, el aumento de la emigración, el aumento de los problemas legales en salud como consecuencia del no respeto hacia otras culturas, el aumento del feminismo y atención al menor en ámbitos de salud y el aumento de la necesidad de atender la multiculturalidad en diferentes contextos ambientales (González, 2006).

Para dar unos cuidados competentes y congruentes culturalmente, el profesional debe respetar las diferencias y similitudes culturales. Leininger entiende a la persona como un ser unido a su cultura, para realizar unos cuidados de calidad hay que respetar y entender dicha cultura. La actitud frente a la enfermedad y las estrategias para luchar contra ella varían de una cultura a otra (González, 2006).

La enfermera debe preservar las acciones que realiza el individuo para mantener su bienestar de acuerdo con su cultura y creencias. En segundo lugar, debe adecuar sus intervenciones a la cultura del paciente para que pueda modificar su estilo de vida y sea eficaz. En tercer lugar, para conseguir que la persona cambie sus conductas, las acciones llevadas a cabo deben ser culturalmente congruentes (González, 2006).

En su Modelo "Sunrise" o Modelo de Sol Naciente describe los componentes de su teoría. Esta teoría sostiene que para poder atender a personas de diferentes culturas es necesario conocer una serie de factores que influyen en ellas, como son, los factores tecnológicos, los factores sociales y familiares, los factores económicos y políticos, los valores culturales y los diferentes estilos de vida. Las decisiones que tomen las personas acerca de su salud estarán influenciadas por estos valores, de aquí la importancia de que el profesional conozca la influencia de ellos (Castillo-Martínez y Fernández-Pujazón, 2015). 
No obstante, la teoría de la Diversidad y Universalidad no es la única teoría que puede ser útil para los enfermeros/as de salud laboral que atienden a un colectivo multicultural. Leininger perteneció a la Escuela del Caring y tuvo como compañera a Jean Watson. Ella desarrolló su teoría del cuidado Transpersonal e identificó diez factores de cuidados que orientan la práctica enfermera. Defiende que el papel de la enfermera es comprender a los individuos y permitir la expresión de los sentimientos pero para que se establezca una relación de cuidado auténtico antes la enfermera debe encontrarse bien consigo misma, comprendiendo y aceptando sus propios sentimientos. Cuando el profesional sanitario reconoce sus propios sentimientos se hace más auténtico a los demás y está preparado para establecer una relación de ayuda ( Raile, 2018).

Por otro lado, Watson considera necesaria la formación en ciencias humanas para poder comprender las características individuales de cada persona porque vivimos en una sociedad multicultural en la cual cada individuo es diferente y único. Debemos permitir la diversidad de creencias y cultura. En este aspecto se relaciona con la teoría de Leininger, fiel defensora de adaptar los cuidados a la cultura de los individuos. Watson también considera necesario adaptar los cuidados enfermeros a las prácticas culturales y las necesidades humanas y sociales. Además, parte de la base de que cuidado no significa cuidar, sino promover la salud de cada individuo teniendo en cuentas sus características individuales y ayudándole a tomar decisiones (Raile, 2018).

Para establecer esta relación de ayuda y confianza, basada en el cuidado y el amor, hay que aceptar la aparición y expresión de sentimientos positivos y negativos. Todo ser humano tiene unas necesidades biofisicas, psicofisicas y psicosociales, y a su vez, todas ellas se ven influenciadas por los factores socioculturales, por lo que será importante conocer cómo son los factores externos e internos 
que influyen en la salud y la enfermedad de cada individuo (Raile, 2018).

Por último, mientras que Leininger considera a la persona con un ser arraigado a su cultura (Castillo-Martínez y Fernández-Pujazón, 2015), Watson lo considera como una unidad de mente, cuerpo y alma, debiendo existir una armonía entre los tres para considerar que la persona goza de salud. De este modo, la enfermedad no siempre va acompañada por pérdida de lo fisico sino por la pérdida de la armonía, es decir, la enfermedad puede ser algo subjetivo (Raile, 2018).

En la figura 1, se expone el mapa de contenidos que se ha llevado a cabo durante el CIMA.

Jornadas de Formación e Innovación Docente del Profesorado | № 2 (2019) Esta obra se distribuye con la licencia Creative Commons 


\section{ROCíO DE DIEGO CORDERO}

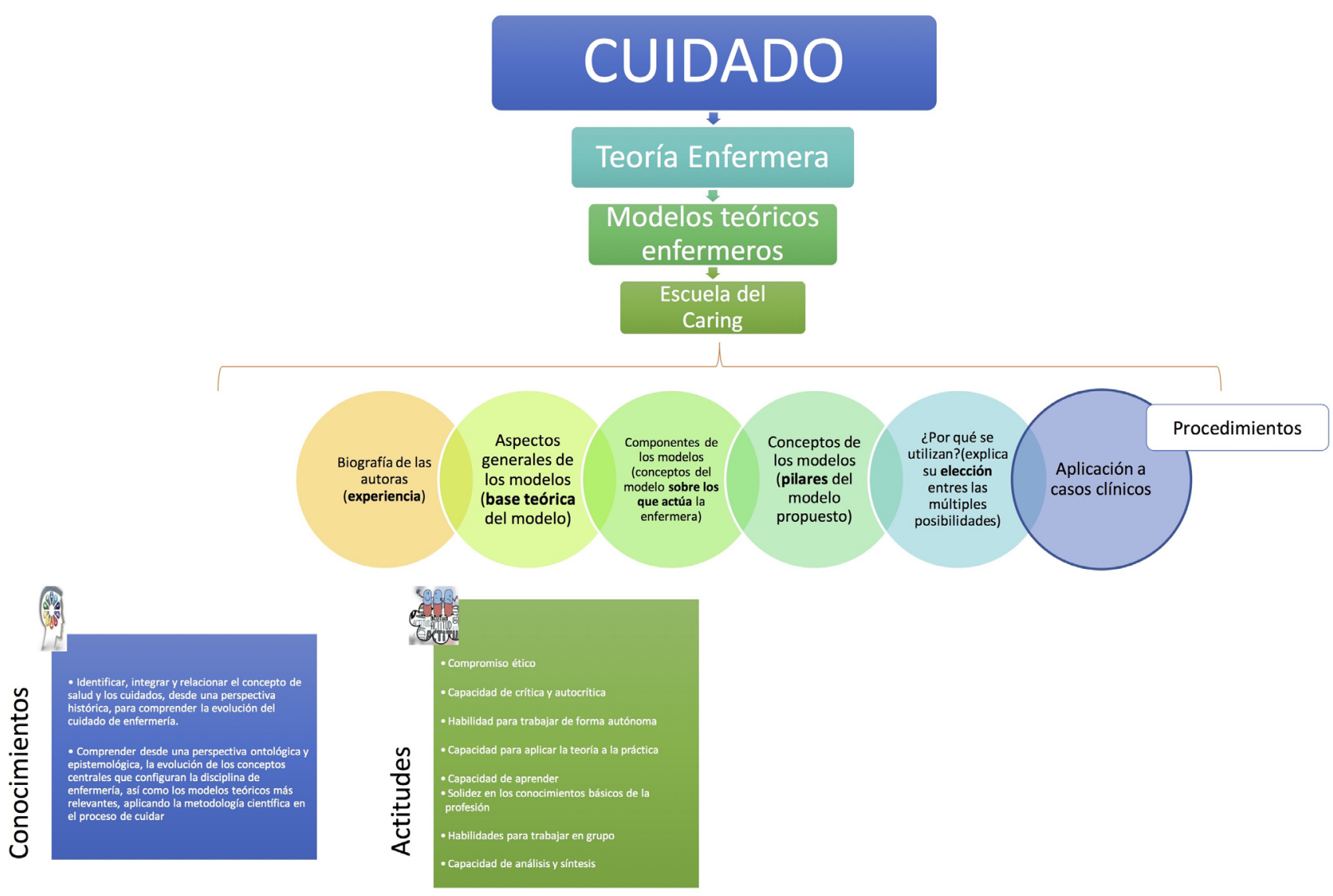

Figura 1: Mapa de contenidos.

Jornadas de Formación e Innovación Docente del Profesorado I № 2 (2019)

(c) (i) $\ominus$ Esta obra se distribuye con la licencia Creative Commons

Internacional (CC BY-NC-ND 4.0.) 


\section{Modelo metodológico posible y secuencia de actividades programada}

El modelo metodológico ideal debería ser totalmente práctico, esto es, reproducir la práctica habitual de abordaje y valoración enfermera. Según este enfoque, la asignatura debería estar más unida a la atención real enfermera, siendo el profesor un guía, orientador y asesor del proceso. La principal ventaja de este enfoque sería la aplicación directa a la realidad, lo que redundaría en un mejor aprendizaje por parte del alumnado. Las principales dificultades que tiene este planteamiento son:

- Imposibilidad del alumnado para aplicar a la práctica clínica los conocimientos teóricos previamente adquiridos, ya que en el primer año de grado no realizan prácticas clínicas.

- Dificultad del profesor para evaluar la adecuación del modelo teórico a los casos clínicos reales.

Por todo ello, el modelo metodológico ideal previamente expuesto requiere ser modificado, generándose el modelo metodológico posible.

En la figura 2 se presenta el modelo metodológico previo, y en tabla 1 se expone la secuencia de actividades del CIMA.

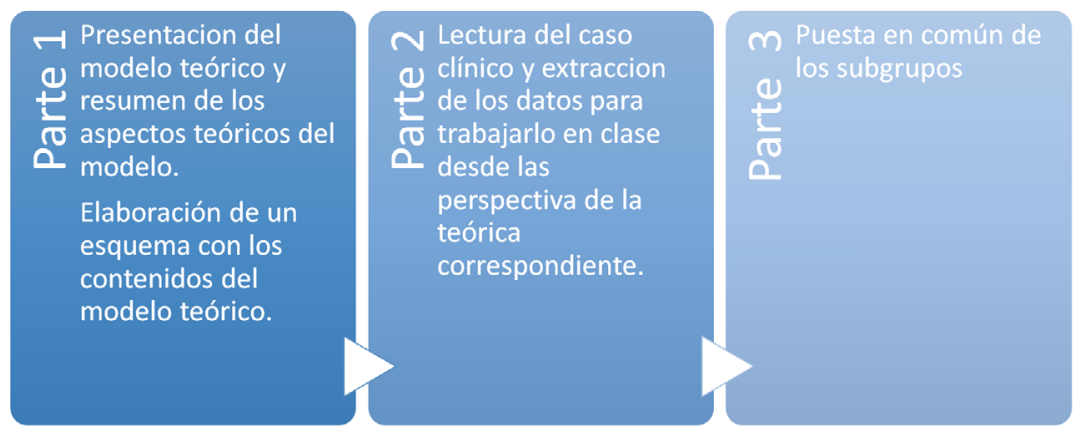

Figura 2: modelo meteorológico previo

En la figura 3 se presenta el modelo metodológico llevado a cabo en el CIMA. 

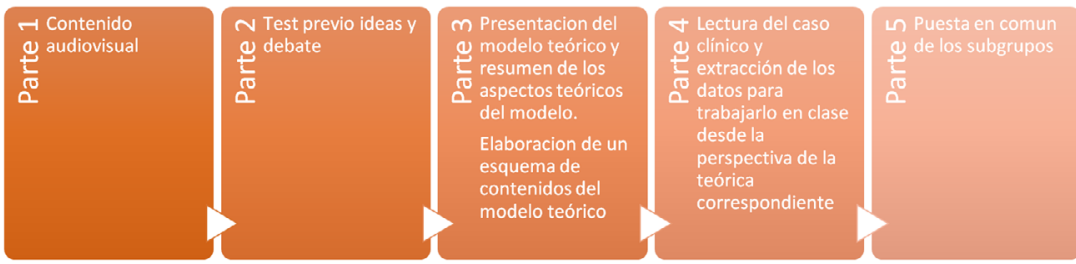

Figura 3: modelo meteorológico del CIMA.

En cuanto a la secuencia de actividades, esta se ha llevado a cabo en tres partes:

Parte 1: Presentación del modelo teórico y resumen de los aspectos más relevantes de su modelo (15 min. aprox.). La propuesta del primer ciclo de mejora fue incorporada en la primera parte, en la que se le pidió a cada subgrupo que elaborase un esquema con los principales conceptos del modelo teórico estudiado, sustituyendo al resumen de los aspectos relevantes que hacía el/la docente. Después, al azar, un componente del subgrupo elegido, salió a la pizarra y reprodujo el esquema elaborado, explicándolo.

Parte 2: Lectura del caso clínico y extracción de los datos para trabajarlo desde la perspectiva de la teórica correspondiente (45-60 min. aprox.)

Parte 3: Puesta en común de los tres subgrupos (45-60 min. aprox.)

En la tabla 1 se expone la secuencia de actividades del CIMA.

Tabla 1: Secuencia de actividades del CIMA.

\begin{tabular}{|l|c|l|}
\hline Fecha & $\begin{array}{c}\text { Tiempo } \\
\text { (horas) }\end{array}$ & \multicolumn{1}{c|}{ Actividades } \\
\hline $13 / 5 / 2019$ & 1 & Contenido audiovisual \\
\hline $20 / 5 / 2019$ & 2 & $\begin{array}{l}\text { Cuestionario de conocimientos previos y tra- } \\
\text { tamiento teórico del tema }\end{array}$ \\
\hline $27 / 5 / 2019$ & 2 & Aplicación a casos clínicos \\
\hline
\end{tabular}

Jornadas de Formación e Innovación Docente del Profesorado | № 2 (2019) Esta obra se distribuye con la licencia Creative Commons Reconocimiento-NoComercial-SinObraDerivada Internacional (CC BY-NC-ND 4.0.) 


\section{Cuestionario inicial-final para hacer un seguimiento de la evolución de los estudiantes.}

Para realizar el seguimiento de la evolución de los modelos mentales de los estudiantes se recurrió a conocer los modelos mentales de partida por parte del alumnado, para esto se propone la elaboración de un cuestionario de conocimientos previos al inicio del CIMA. Este mismo cuestionario se les pasó al final, para ver la evolución de los modelos mentales del alumnado. Esto ha permitido la revisión del planteamiento inicial así como el ajuste de los contenidos a las necesidades reales del alumnado. Para llevar a cabo la evaluación de estos cuestionarios se aplicó una metodología cualitativa con análisis de los las respuesta a las preguntas planteadas.

Unido a ello, el profesorado corrigió las tareas del alumnado donde se vio la aplicación real de lo aprendido a los casos clínicos propuestos, dando respuestas al alumnado que permitan la retroalimentación continua que redunde en el verdadero aprendizaje del alumnado más allá de ser sólo un instrumento para la evaluación. De esta manera:

- Se devuelve al alumnado los casos analizados y comentados semanalmente, haciendo hincapié en los aspectos más relevantes y no en la evaluación.

- Se ofrece la posibilidad de realizar tutorías individuales, que refuercen los conocimientos y mantengan la comunicación bidireccional.

Jornadas de Formación e Innovación Docente del Profesorado | № 2 (2019) Esta obra se distribuye con la licencia Creative Commons 


\section{Aplicación del CIMA}

\section{Relato resumido de las sesiones}

En el curso pasado, se puso el primer ciclo de mejora y se decidió tratar el tema de la Enfermería transcultural (entendiendo que la cultura es un tema que podía despertar el interés del alumnado) introduciéndose un cuestionario de conocimientos previos con:

- Preguntas: Cuál es tu cultura? /¿Qué otras culturas conoces? di al menos dos./ ¿Piensas que hay aspectos de la cultura que pueden influir en la salud? Si la respuesta es sí, señala al menos dos./

- Imágenes: observa esta imagen y rellena los bocadillos con lo que pienses pueden estar pensando tanto la enfermera como el paciente en esta situación.

El alumnado que fue adecuadamente informado mostró asombro pero también interés por participa, generándose un buen clima de trabajo en el aula. El papel facilitador y moderador del docente permitió tratar los aspectos de una manera mucho más eficaz y el recapacitar desde lo propio hizo que mejorase la comprensión general.

Jornadas de Formación e Innovación Docente del Profesorado | № 2 (2019) Esta obra se distribuye con la licencia Creative Commons Reconocimiento-NoComercial-SinObraDerivada 4.0 Internacional (CC BY-NC-ND 4.0.) 
Test exploración de ideas previas

1. ¿Qué es la cultura?

2. ¿Cuál es tu cultura?

3. ¿Qué otras culturas conoces? di al menos 2.

4. ¿Piensas que hay aspectos de la cultura que pueden influir en la salud? Si la respuesta es si, señala al menos 2 .

5. Observa esta imagen y rellena los bocadillos con lo que pienses pueden estar pensando tanto la enfermera como el paciente en esta situación.

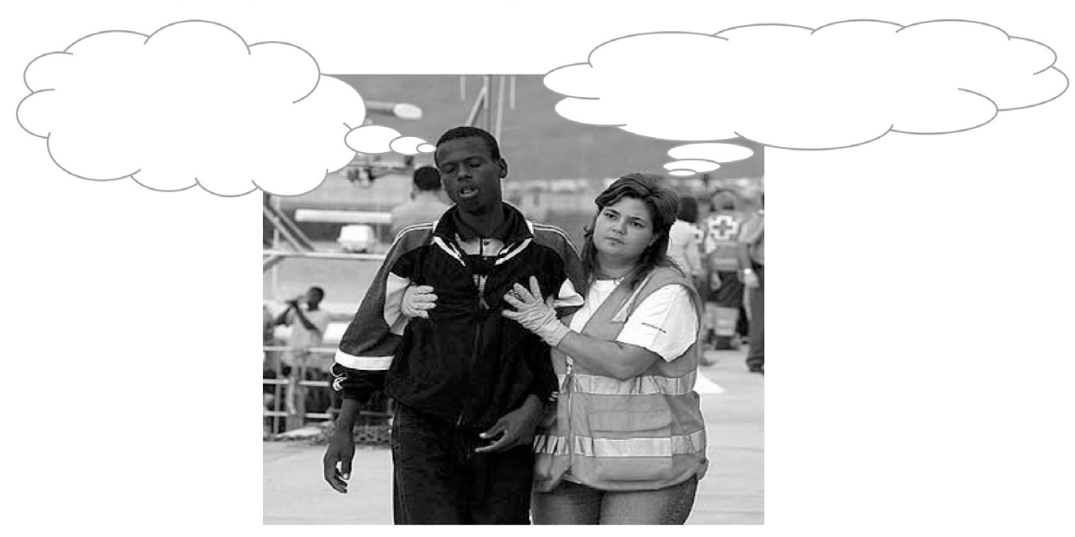

Figura 4: Cuestionario de ideas previas.

En este curso al CIMA se incorpora lo planteado en el curso pasado que fue de 4 horas, incrementando una hora, en la que trató con contenido audiovisual los conceptos de los cuidados culturales, que completó lo realizado en el CIMA del curso anterior.

Al tratarse de contenido audiovisual el alumnado se muestra interesado; además apoyar con imágenes y movimientos los conceptos ayudó mucho a la comprensión

Jornadas de Formación e Innovación Docente del Profesorado | № 2 (2019) Esta obra se distribuye con la licencia Creative Commons Reconocimiento-NoComercial-SinObraDerivada 
del model o teórico. El alumnado consideró de gran ayuda comenzar con este apoyo inicial que sirvió como primera toma de contacto e introducción al modelo teórico posterior.

\section{Evaluación del diseño y de la intervención}

Para saber cómo están funcionando las clases englobadas en el CIMA propuesto en el presente documento y posibilitar el ajuste de las actividades diseñadas se han identificado los siguientes recursos:

- Notas de campo: se tomaron in situ durante el desarrollo del CIMA.

- Diario del profesor: se dedicó semanalmente unos minutos a escribir sobre el desarrollo de la experiencia para tomar conciencia del propio funcionamiento del CIMA y de la intervención, para incluso poder introducir mejoras sobre la marcha.

- Encuesta de opinión al final del CIMA que permita al alumnado proponer su punto de vista así como posibles mejoras.

\section{Resultados de la evaluación}

Tras llevar a cabo el análisis de los cuestionarios, la totalidad reconoce su propia cultura, reconoce otras culturas, cree que hay elementos culturales que pueden afectar a la salud y reconoce la importancia de trabajar desde la perspectiva culturalmente competente.

Tras esto, se le entregan casos clínicos en los que deben aplicar lo comentado en el cuestionario.

Se establecen por tanto 3 modelos de respuesta:

A. Reconoce su propia cultura; reconoce otras culturas; cree que sin duda hay elementos culturales 
que pueden afectar a la salud. Reconoce la importancia de trabajar desde la perspectiva culturalmente competente. Lo aplica al caso clínico.

B. Reconoce su propia cultura; reconoce otras culturas; cree que hay elementos culturales que pueden afectar a la salud. Reconoce la importancia de trabajar desde la perspectiva culturalmente competente. NO lo aplica al caso clínico.

C. Reconoce su propia cultura; reconoce otras culturas; cree que puede haber elementos culturales que pueden afectar a la salud. No reconoce la importancia de trabajar desde la perspectiva culturalmente competente. NO lo aplica al caso clínico.

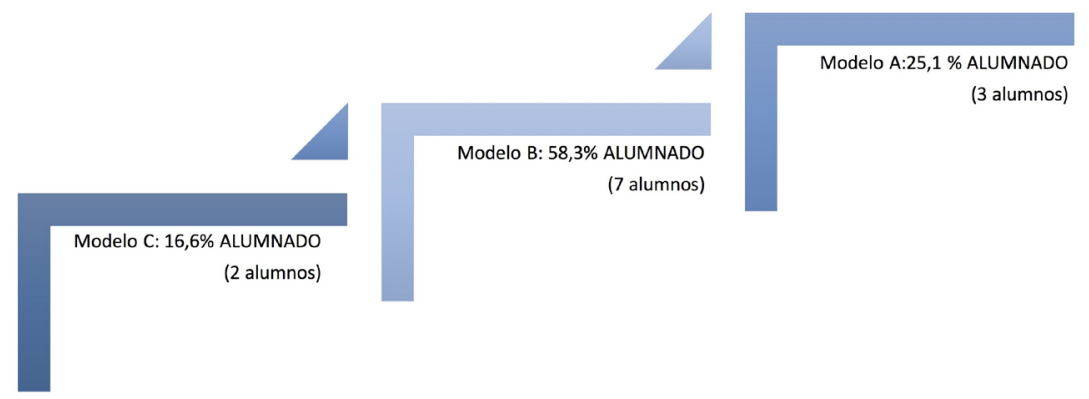

Figura 5: Escalera de aprendizaje. Elaboración propia.

Tras analizar las notas de campo tomadas durante las sesiones, el diario del profesor y la corrección de los casos clínicos donde aplican el modelo teórico, los análisis muestran unos muy buenos resultados; la incorporación de contenido audiovisual previo en el que el alumnado ha comprendido más y mejor el modelo teórico junto con el análisis de los conceptos de cultura que empiezan por el reconocimiento de las barreras propias ha hecho que la evolución del aprendizaje haya sido satisfactoria, estando el $100 \%$ del alumnado en modelo de respuesta $A$.

Jornadas de Formación e Innovación Docente del Profesorado | № 2 (2019) Esta obra se distribuye con la licencia Creative Commons Reconocimiento-NoComercial-SinObraDerivada 


\section{Evaluación del CIMA}

\section{Cuestiones a mantener y cambios a introducir para un futuro CIMA}

Respecto a las cuestiones a mantener y cambios a introducir para un futuro CIMA más amplio (en el mapa de contenidos y problemas, en la metodología y actividades, en el sistema de evaluación...), se considera apropiado mantener la estructura metodológica puesta en marcha en los dos CIMA e intentar exportarla a otros contenidos de la asignatura. También se estima oportuno continuar contando con herramientas de evaluación como el diario del profesor, las notas de campo y cuestionario de evaluación final que permiten un abordaje mucho más global del alumnado.

\section{Aspectos de la experiencia a incorporar en la práctica docente habitual}

En lo referido a los aspectos de la experiencia que se pretenden incorporar a toda la práctica docente habitual, resaltar el importante papel de la evaluación previa de los conocimientos y actitudes del alumnado para que éste sea el punto de partida.

\section{Principios Didácticos}

Finalmente, los principios didácticos argumentados que han guiado la experiencia (Porlán et al. 2017), y que deben permanecer en el futuro son:

- Diseñar previamente a cada modelo teórico enfermero un cuestionario de ideas previas para poder adaptar los contenidos al nivel de conocimiento del 
alumnado, conocer los puntos de interés y atender a la diversidad.

- Elaboración de mapas conceptuales previos que ayuden a ordenar y jerarquizar los contenidos a impartir.

- Uso de temas de actualidad o alguna pregunta que despierte el interés del alumnado a la vez que fomente la actitud crítica y analítica.

- Realización de notas a modo de "diario del profesor" que ayude a la reflexión diaria del desarrollo de las sesiones.

- Utilizar el modelo de "dar clase con la boca cerrada" (Finkel, 2008) en el que el alumnado será el protagonista, siendo sólo el docente un facilitador y un buen gestor del tiempo.

- Implementar la evaluación continua de las tareas desarrolladas en las sesiones, las lecturas realizadas y la resolución de los casos, a través de entregas semanales que permitan el feedback con el alumnado y la corrección de errores, facilitando además el aprendizaje.

- Incluir la autoevaluación del alumnado en la práctica docente, además de la evaluación de la asignatura (de la parte correspondiente al trabajo en Grupos Pequeños) así como del/ de la docente.

Jornadas de Formación e Innovación Docente del Profesorado | № 2 (2019) Esta obra se distribuye con la licencia Creative Commons 


\section{Referencias bibliográficas}

Castillo-Martínez, A. y Fernández-Pujazón, R. (2015). Enfermería Transcultural en Salud Laboral. Revista Enfermería del Trabajo, 5 (3), 82-89. Recuperado de https:// dialnet.unirioja.es/servlet/articulo? codigo $=5213012$.

Finkel, D. (2008). Dar clases con la boca cerrada. Publicaciones Universidad de Valencia.

González, D. L. (2006). Buscando un modelo de cuidados de enfermería para un entorno multicultural. Gazeta de Antropologia, 22 (32). Recuperado de http://hdl. handle.net/10481/7118

Izquierdo, E. (2015) Enfermería: Teoría de Jean Watson y la inteligencia emocional, una visión humana. Revista Cubana Enfermería, 31 (3). Recuperado de http: / / www.revenfermeria.sld.cu/index.php/enf/article/view/686/131

Porlán, R. (Coord), Vázquez, J., Solís, E., Martín, R., Pineda, J. A., Duarte, O., De Alba, N., García, E., Navarro, E., Rivero, A., García, F. F., Feria, B., Guerra-Martín, M. D., Fuentes, A., Mora, J. P. y Herrera, J. A. (2017). Enseñanza universitaria. Cómo mejorarla. Ediciones Morata. Sevilla.

Jornadas de Formación e Innovación Docente del Profesorado | № 2 (2019) Esta obra se distribuye con la licencia Creative Commons 